\title{
Relation between Knowledge and Anxiety Level of Patients Undergoing Upper Gastrointestinal Endoscopy at Tanta University Hospital
}

\author{
Sara A. Anwar ${ }^{1}$, Afaf Abdelaziz Basal ${ }^{2}$, Mahmoud F. Selim ${ }^{3}$ \\ and ${ }^{4}$ Heba A. Al-Metyazidy ${ }^{4}$.
}

\footnotetext{
${ }^{1}$ Baccalaureate Degree in Nursing Science, Faculty of Nursing, Tanta University, Egypt.

${ }^{2}$ Assist. prof. of Medical Surgical Nursing, Medical Surgical Nursing Department, Faculty of Nursing, Tanta University, Egypt.

${ }^{3}$ Professor of Medical and Liver disease, Medical Disease Department, Faculty of Medicine, Tanta University, Egypt.

${ }^{4}$ Lecturer of Critical Care Nursing, Medical Surgical Nursing Department, Faculty of Nursing, Tanta University, Egypt.

E-mail: ${ }^{1}$ saraanwar008@gmail.com, ${ }^{2}$ drafafbasal@yahoo.com . roka-omarali@yahoo.com,
}

\begin{abstract}
Upper gastrointestinal endoscopy plays a major role in diagnosis and management of upper gastrointestinal disorders. Lack of patient's knowledge regarding endoscopy procedure is the main reason for increasing anxiety. This study aimed to: Assess knowledge and anxiety levels of patients undergoing upper gastrointestinal endoscopy. The study was carried out: In the gastrointestinal endoscopy units, Tanta University Hospital: A convenience sample of 150 adult patients with GI disorders admitted to GI endoscopy units at Tanta University Hospital and scheduled for GI endoscopy were recruit to the study. The research questions include, 1- What are the levels of knowledge of patients undergoing upper GI endoscopy at Tanta University Hospital? 2- What are relations between levels of state and trait anxiety and different variables? Tools of the study: Two tools were used, Tool (I) Structured interview questionnaire: This tool was developed and used by the researcher for collection of baseline data and is consisted of three parts: Socio demographic data, patient's clinical information and patient's knowledge regarding upper gastrointestinal endoscopy. Tool (II) The State Trait Anxiety Inventory Scale: The original scale was developed by Spielberger in 1970 to evaluate respectively, state and trait anxiety. Results: It was observed that Less than half $(44.00 \%)$ of studied patients had poor knowledge score, while $(45.33 \%)$ had severe state anxiety and more than half $(51.33 \%)$ had severe trait anxiety. there was a high negative significant correlation $(r=-0.256,-0.318$ respectively) between knowledge and The State Trait Anxiety Inventory Scale. Conclusion: Providing information to the patient before upper gastrointestinal endoscopy, decrease their level of anxiety and improve tolerance level during endoscopy procedure. Recommendations: It was recommended that provision of institutional written policies and guidelines regarding increasing knowledge and declining anxiety for patients undergoing upper gastrointestinal endoscopy.
\end{abstract}

Key words: Upper gastrointestinal endoscopy, knowledge, anxiety. 


\section{Introduction:}

Upper gastrointestinal (GI) endoscopy is a safe and widely used procedure. Furthermore, endoscopy surpassed its function as an examination tool and it consider a rapid and efficient therapeutic tool with low invasiveness. However, the improvement in the endoscopy procedure increase the image quality, the most important component affecting the quality of the procedure is patient compliance. One of the most important factors that impact patient compliance is the patient's anxiety level before the procedure ${ }^{(1)}$.

Globally, 750 gastroscopies surgery per 100.000 populations are subjected in 2004 . Approximately 3.5 million people underwent gastroscopy in United States in 2016. The most common indications of upper GI endoscopy in Egypt include esophageal varices which represent 54\%, upper GI erosions $26 \%$, peptic ulcer $8 \%$, esophagitis $6 \%$ and upper GI tumors $6 \%$ respectively of patients admitted to GI endoscopy units in 2010 . The yearly report of gastrointestinal endoscopy center at Tanta University Hospital Center indicated 11.000 cases of GI endoscopy referred to the center in $2017^{(2-4)}$.

Upper gastrointestinal endoscopy is used to examine esophagus and upper gastrointestinal tract (UGIT). It is considered the investigation of choice in cases of dyspepsia, reflux symptoms or alarm symptoms and is mandatory for a precise diagnosis in cases of these upper abdominal complains ${ }^{(5)}$.

Upper gastrointestinal endoscopy procedure usually done for the purpose of diagnosing any upper GI problems and usually create anxiety for patient. Anxiety in patient occurs due to lack of awareness about the procedure and fear of discomfort or pain in procedure throughout ${ }^{(6-8)}$.

Anxiety is a feeling of fear, uneasiness and worry. Usually generalized and unfocused, it is often accompanied by muscular tension, restlessness and problems in concentration $^{(9)}$.

Upper gastrointestinal endoscopy nurses play a critical role in the provision of safe, high quality preparation or care for patients undergoing upper GI endoscopy such as preparing the endoscopic room with the right instrument and necessary devices for examination of the upper $\operatorname{GIT}^{(10,11)}$.

Moreover, the nurse offers a holistic package of care to patients undergoing upper GI endoscopy, encompassing the psychological, physiological and sociological needs of the patient. Also, the nurse already has the skills and knowledge to assess the needs of each individual attending for endoscopy from admission to 
discharge ${ }^{(12,13)}$.

In addition to, providing information to the patient before upper GI endoscopy may decrease the level of anxiety and improve tolerance level of patient during endoscopy procedure. To ensure the quality and safety of the procedure in upper GI endoscopy, it is important to know the patient anxiety level and the effective factors to reduce it, as enhance patient knowledge related to procedure $^{(14-19)}$.

Assessment is the first step of the nurse and patient interaction. It is also the most important aspect of the pre and post upper GI endoscopy procedure. In addition, to ensure individuality of care by finding out what the patient want and need to know. Also, help in clarify misconceptions and allow patient to express feeling ${ }^{(20)}$.

The pre procedure nursing assessment of the patient includes reviewing of medical history of the patient regarding allergy to any medicines, history of taking any medication, surgery and radiation treatments to esophagus, stomach or duodenum. Also, the nurse asks the patient taking nothing by mouth for about six hours, changing into a loose fitting gown, intravenous access be established, spectacles and dentures are removed and the informed consent sign by the patient and put in the patient record ${ }^{(21,22)}$.
The intra procedure nursing assessment includes frequent check and records all vital signs, and put the patient in appropriate position for performing the procedure usually the left side. A mouth guard place in the mouth to protect the patient teeth from the endoscopy. Then the lubricate tip of the endoscopy guide smoothly and slowly into the mouth and down into the esophagus, additionally the endoscopy nursing staff asks the patient to swallow to assist in moving the tube along ${ }^{(23)}$.

The post procedure nursing assessment includes frequent check and records all vital signs, assesses patient's response to procedure as nausea, bloating, level of consciousness, ability to swallow and move extremities. If the patient's throat numb after the procedure, the patient doesn't eat or drink until throat is no longer numb, and gag reflex return to normal ${ }^{(24)}$.

It is also crucial that, the nurse gives the right information before the procedure to relieve patient's anxiety. During the procedure the nurse must help the endoscopist. After the completion of the procedure, the nurse manages the endoscopic finding safely as biopsies ${ }^{(25) .}$

\section{Subjects \& Method:}

\section{Subjects:}

The aim of this study is to assess knowledge and anxiety levels of patients 
undergoing upper GI endoscopy at Tanta

University Hospital.

\section{Research questions:}

- What are the levels of knowledge of patients undergoing upper GI endoscopy at Tanta University Hospital?

- What are relations between levels of state and trait anxiety and different variables?

\section{Study design:}

Descriptive and exploratory study design was utilized to achieve the aim of the study and answer the research questions.

\section{Setting of the study:}

This study was conducted in the gastrointestinal endoscopy units at Tanta University Hospital.

\section{Subjects:}

A convenience sample of 150 adult patients with GI disorders admitted to GI endoscopy units at Tanta University Hospital and scheduled for GI endoscopy were recruit to the study. The subject size of participants was calculated using a power analysis.

\section{The inclusion criteria were as follow:}

- Ages ranged from 18 to 60 years.

- Both sexes.

- Conscious patients.

- First time undergoing upper GI endoscopy.

\section{The exclusion criteria were as follow:}

- Patients taking anti-anxiety drugs in the last $72 \mathrm{~h}$.

- Patients unable to communicate.

- Patients with dementia or psychiatric disorders.

- Patient in emergency condition.

\section{Data collection tools:}

Two tools were used in this study to collect pertinent data related to the aim as follow:

Tool (I): $\quad$ Structured Interview Questionnaire.

Tool (II): The State Trait Anxiety Inventory Scale (STAI).

Tool (I): Structured Interview Questionnaire:

This tool was developed by the researcher after review of the relevant literature to assess patient's socio demographic data, clinical information and their knowledge about upper GI endoscopy. It consisted of three parts as follow:

\section{Part 1: Socio demographic data: ${ }^{(1-3)}$}

It was developed to assess patient's socio demographic data that covered the following variables: age, sex, marital status, place of residence, socioeconomic status, level of education, income level and occupation.

Part 2: Patient's clinical information: ${ }^{(9,21,22)}$

This part was consisted of statements that used to assess patients information about their health history, it was comprise the following areas: present diagnosis, past 
medical history, history of hospitalization, the last laboratory studies, heart rate, respiration rate, blood pressure, associated chronic diseases, mobility status, past and current intake of medication, the last attack of bleeding, previous time of bleeding factors that participate in bleeding such as certain drug, smoking, lifting heavy objects, constipation, coughing or sneezing.

Part 3: Patient's knowledge assessment: (26-27)

This part was developed to assess patient's knowledge about upper GI endoscopy such as: Definition, indication, purposes, risk factors, procedure, possible occurrence of discomfort or side effect, the place where the procedure was performed, investigation was done, type of anesthesia used, physical and psychological preparation before procedure. Also, what was actually happen during the procedure, recovery period and discharge instruction such as follow up plan, types of activities, return to work, types of diet, medication schedule, hygiene care, signs of complication and referral places.

Scoring system of patient's knowledge assessment questionnaire was done as follow:

Patient's knowledge assessment questionnaire was consisted of 22 statements about knowledge and information of the patients regarding upper GI endoscopy. Each question has a number of a group of answer points.

Scoring system: Patient who was responded by correct and complete answer was given a score two, correct and incomplete answer was given a score one and the patients who responded wrong and not answer was given a score zero. Less than $60 \%$ of total score was considered as poor, from $60 \%$ to less than $75 \%$ was considered as fair, and from $75 \%$ and more was considered as good.

\section{Tool (II): The State Trait Anxiety} Inventory Scale (STAI) ${ }^{(28-31)}$.

The original scale the State Trait Anxiety Inventory was developed by Spielberger in $\mathbf{1 9 7 0}^{(28)}$. It was used to evaluate respectively, state and trait anxiety. The SAI Scale determines how the individual feels at a particular moment and under certain circumstances. The scale is designed in quadruple Likert-type scale varying between never, sometimes, usually and almost always. The TAI Scale determines how the individual generally feels, independent of the status and the conditions which surround him/her.

Scoring system: Both the SAI and TAI Scales comprised of 20 items each one 


\section{Tanta Scientific Nursing Journal}

scored on 4 point forced choice Likert type response scales. Scores ranged from 20 to 80 , with normal scores or no anxiety score 20, low scores from 21-40 suggest mild anxiety, median scores from 41-60 suggest moderate anxiety, while high scores from 61-80 suggest severe anxiety.

\section{Method:}

\section{1- Administrative process:}

Approval was obtained from the responsible authority of GI endoscopy units at Tanta University Hospitals before conducting the study.

\section{2- Ethical consideration:}

- Written consent was obtained from every patient included in the study after explanation of the aim of the study and assuring them of confidentiality of collected data.

- Confidentiality and anonymity was maintained by the use of code number instead of name and the right of withdrawal is reserved.

- Privacy was assured to the patients.

\section{3-Tools development:}

- Tools I (Structured Interview Questionnaire) was developed by researcher based on relevant literature review for collection of baseline data ${ }^{(1,3,9}$, $21,22,26,27)$

- Tool II (The STAI Scale) was developed by Spielberger in $\mathbf{1 9 7 0}^{(28)}$, and were initially designed as a single instrument to measure both anxiety trait and anxiety state. Arabic translation of this tool was done by El-Behairy in $\mathbf{1 9 8 4}^{(29)}$ to a certain relevance and completeness.

\section{4- Content validity:}

- All tools were tested for content validity by a nine jury of experts in the field of Medical Surgical Nursing, Critical Care Nursing at the Faculty of Nursing and Medical specialists, and also Biostatistics at the Faculty of Medicine.

- Modifications were done to certain relevance and completeness.

\section{5-Reliability of the tools:}

All tools of the study were tested for reliability and Cronbach alpha was used and found to be 0.88 for Tool I and 0.98 for Tool II, which consider highly reliable tools.

\section{6- A pilot study:}

- A pilot study was carried out on a sample of 150 patients from GI endoscopy unit in order to test clarity, feasibility and applicability of the tools. Subject of pilot study was excluded from the original sample.

\section{7- Data collection:}

A. Data collection was conducted over a period of 6 months (started from first of March to the end of September 2017). Data was collected during the morning shift 
according to Tanta University Hospital rules, in the waiting room before the procedure of upper GI endoscopy. About 5 to 10 patients were interviewed daily from 8:30 Am to 10:30 Am, through two days / week.

B. The selected patients who met the inclusive criteria were asked to participate in the study after establishing trusting relationship and explaining the aim of the study. After that all patients provided written informed consent for participation in the study. Then data was collected during interview. Each patient were reassured that, they obtained information will be confidential and used only for the purpose of the study.

C. The interview questionnaire sheet information and STAI Scale questions were given to the participants by the researcher (After translation by experts into Arabic and was tested for content validity and clarity), and all patients were asked to answer on all questions in the interview questionnaire sheet individually that was previously explained by the researcher.

D. Assessment of studied patients was presented in two stages according to the following sequence:

1. Stage one (Structured Interview Questionnaire): To assess socio demographic data, patient's clinical information and patient's knowledge regarding upper GI endoscopy.

2. Stage two (The STAI Scale): To assess patient's anxiety level.

1. In stage one, each patient was assessed regarding socio demographic data of the patients (age, sex, marital status, place of residence, socioeconomic status, level of education, income level and occupation), their clinical information (present diagnosis, past medical history, history of hospitalization, the last laboratory studies, heart rate, respiration rate, blood pressure, associated chronic diseases, mobility status, past and current intake of medication).

Moreover, patient's knowledge about upper GI endoscopy was assessed such as definition, indication, purposes, risk factors, procedure, possible occurrence of discomfort or side effect, the place where the procedure was performed, investigation was done, type of anesthesia used, physical and psychological preparation before procedure. Also, what was actually happen during the procedure, recovery period and discharge instruction. It takes about 20-30 min to answer.

2. In stage two, the anxiety level was assessed through the STAI Scale. It is a two part 40 items self-report. The state portion (20 items) measures how a person feels at 


\section{Tanta Scientific Nursing Journal}

the time of the endoscopy procedure and the trait portion (20 items) measures a person's general disposition. This scale is simple to answer, the state portion given to studied patients at first and then trait portion given, generally taking $<10 \mathrm{~min}$ to complete and easy to score.

E. The researcher was available in GI endoscopy unit for any expectations and checking each question after complete to be sure that all questions were answered. (Each interview duration ranged from 30 to 40minutes).

F. In the event of no answer, patients were further asked whether or not they wished to receive information about this specific item. On other hand, in the event of positive answer, they were discussed about their knowledge and from whom did they get the knowledge from.

G. After data collection, data was coded, analyzed then tabulated under the direction of a statistician to obtain results to answer the research questions.

H. Finally, most patients approach an endoscopic procedure with fear and anxiety. Moreover, to lessen or even prevent this, providing patients with information is essential in order to prepare the patients physically, emotionally and intellectually for the procedure of upper GI endoscopy.

\section{Statistical analysis:}

After completion of data collection, each sheet of interview questionnaire sheet information and STAI Scale questions was coded, organized and categorized then the data was tabulated and presented into frequency distribution tables.

The following tests used in the study were chi square test and $\mathrm{P}$-value to assess knowledge and anxiety levels of patients undergoing upper GI endoscopy at Tanta University Hospital. The data was collected and statistically analyzed using the Statistical Package for Social Sciences (SPSS) version 20 for continuous variables (mean $\pm \mathrm{SD}$ ), ANOVA t tests were used for the detection of significant differences for studied patients. Pearson's correlation coefficient was used to test correlation between variables.

10. The level of significance chose in the study was set at 0.05 levels.

Non significance if P-value $>0.05$

Significance if $\mathrm{P}$-value $<0.05$

High significance if $\mathrm{P}$-value $<0.001$

Results:

Table (1) illustrates percentage distribution of studied patients according to socio demographic characteristics. This table showed that the mean age of studied patients was $(52.56 \pm 12.07)$. More than half $(60.67 \%)$ in the age group ranged 
from 51 to 60 years old, while $(15.33 \%)$ in the age group ranged from 30 to 40 years old.

In relation to sex, more than two thirds $(71.33 \%)$ of studied patients were male, while less than one third $(28.67 \%)$ were female.

As regards to marital status, more than three quarters $(82.67 \%)$ of studied patients were married, while $(4.00 \%)$ were divorced.

In relation to residence, less than three fourths $(72.00 \%)$ of studied patients have lived in rural areas, while less than one third $(28.00 \%)$ have lived in urban areas.

As regards to income level, less than two thirds $(62.67 \%)$ of studied patients had low income level, while (12.00\%) had high income level.

In relation to level of education, more than one half $(54.00 \%)$ of studied patients were illiterate, while $(6.67 \%)$ had secondary education.

As regards to occupation, less than two thirds $(64.00 \%)$ of studied patients had no work, while more than one third $(36.00 \%)$ working.

Table (2) shows distribution of studied patients according to current diagnosis and past medical history. This table illustrated that less than half $(44.67 \%)$ of studied patients referred from other department to endoscopy units, while, $(37.33 \%)$ and $(18.00 \%)$ of studied patients were admitted from outpatients and emergency departments respectively.

In relation to current diagnosis, It can be seen that the most common diagnosis of studied patients who undergoing upper GI endoscopy was esophageal varices and ulcer $(28.67 \%)$, while the least diagnosis was abdominal pain and distention (5.33\%).

As regards to past medical history, more than one third $(36.00 \%)$ of studied patients had past medical history of liver and spleen diseases, while $(2.00 \%)$ had past medical history of cancer.

Finally, regarding history of hospitalization, more than half $(54.67 \%)$ of studied patients had no previous history of hospitalization, while less than half $(45.33 \%)$ had previous history of hospitalization and nearly two thirds (63.24\%) hospitalized for medical reasons.

Table (3) illustrates distribution of studied patients according to history of upper gastrointestinal bleeding. One can notice that, more than half $(57.03 \%)$ of studied patients had information about upper GI endoscopy from medical team, while $(28.13 \%$ and $23.44 \%)$ of studied patients had their information from friends and media respectively.

As regards to factors that participate 
bleeding less than two thirds $(62.07 \%)$ of studied patients had no factors participate in bleeding, while (37.93\%) had factors that participate bleeding. The most common precipitating factors of bleeding was coughing or sneezing $(36.36 \%)$, while the least one was constipation (15.91\%).

Figure (1): Percentage distribution of studied patients in relation to their total knowledge score regarding upper gastrointestinal endoscopy.

This figure showed that less than half $(44.00 \%)$ of studied patients had poor knowledge score regarding upper GI endoscopy, while $(24.00 \%$ and $32.00 \%)$ of studied patients had fair and good knowledge score respectively.

Table (4) illustrates percentage distribution of the studied patients in relation to their total Anxiety Inventory Scale pre upper gastrointestinal endoscopy procedure. It can be seen that less than half $(45.33 \%)$ of studied patients had severe state anxiety, while (18.67\%, $10.00 \%$ and $26.00 \%$ ) had normal, low and moderate state anxiety respectively.

Regarding total Anxiety Trait Scale, more than half $(51.33 \%)$ of studied patients had severe trait anxiety, while $(12.00 \%, 16.00 \%$ and $20.67 \%)$ had normal, low and moderate trait anxiety respectively.
It was found that, highly statistical significant difference in relation to patients total anxiety state and total anxiety trait pre upper GI endoscopy procedure.

Table (5) reveals relation between patient's total knowledge score and The State Anxiety Inventory Scale. It can be seen that, $(50.00 \%)$ of studied patients had good knowledge and normal state anxiety scale, while more than half $(58.82 \%)$ of studied patients had poor knowledge and severe state anxiety scale.

Regarding relation between total knowledge score and The Trait Anxiety Scale, half (50.00\%) of studied patients had good knowledge and normal trait anxiety scale, while more than half $(59.74 \%)$ of studied patients had poor knowledge and severe trait anxiety scale.

It can be seen that, there was a statistical significant difference among studied patients in relation to knowledge and The Anxiety Inventory Scale, p-value was $<0.05$.

Table (6) reveals relation between socio demographic data of patients and their total knowledge score. This table showed that the mean age of studied patients who had good knowledge was (52.18 \pm 8.35$)$.

In relation to sex, less than one third $(31.33 \%)$ of studied patients who had poor knowledge were male, while (12.67\%) were 
female. On other hands, more than one fifth $(20.67 \%)$ of studied patients who had good knowledge were male.

As regards to marital status, nearly one third $(32.67 \%)$ of studied patients who had poor knowledge were married. While, (26.67\%) who had good knowledge were married.

In relation to residence, more than one third $(34.67 \%)$ of studied patients who came from rural had poor knowledge, compared to $(9.33 \%)$ came from urban. While, $(21.33 \%)$ of studied patients who came from rural had good knowledge.

In relation to level of education, more than one quarter $(25.33 \%)$ of studied patients who had poor knowledge were illiterate, while $(2.00 \%)$ were graduated from university. On the other hand, (13.33\%) of studied patients who had good knowledge were illiterate, while $(8.00 \%)$ were graduated from university.

It can be seen that, there was a highly statistical significant difference between knowledge in relation to sex, marital status, socioeconomic status, income level and occupation, $\mathrm{p}$-value was $<0.05^{*}$

Table (7) Illustrates relation between socio demographic data of patients and The State Anxiety Inventory Scale. This table showed that the mean age of studied patients who had severe state anxiety was
$(51.67 \pm 8.51)$.

In relation to sex, less than one third $(28.67 \%)$ of studied patients who had severe state anxiety were male, while (16.67\%) were female. On other hand, $(7.33 \%)$ of studied patients who had low state anxiety were male, while (2.67\%) were female.

As regards to marital status, more than one third $(36.67 \%)$ of studied patients who had severe state anxiety were married, while $(2.67 \%)$ were single. On other hand, $(8.00 \%)$ of studied patients who had low state anxiety were married, while $(2.00 \%)$ were single.

In relation to residence, the percentage $(33.33 \%$ and $12.00 \%)$ of studied patients who came from rural and urban areas had severe state anxiety respectively, while $(6.00 \%$ and $4.00 \%)$ of studied patients who came from rural and urban had low state anxiety respectively.

In relation to level of education, more than one fifth $(23.33 \%)$ of studied patients who had severe state anxiety were illiterate, while $(2.67 \%)$ were graduated from university. Also, (6.00\%) of studied patients who had low state anxiety were illiterate.

It can be seen that, there was a highly statistical significant difference between the SAI Scale in relation to age, marital status, socioeconomic status and education level, 
p-value was $<0.05^{*}$

\section{Table (8) reveals relation between socio} demographic data of patients and The Trait Anxiety Inventory Scale. This table showed that the mean age of studied patients who had severe trait anxiety was (51.62 \pm 11.58$)$.

In relation to sex, more than one third $(35.33 \%)$ of studied patients who had severe trait anxiety were male, while $(16.00 \%)$ were female. On the other hand, $(12.00 \%)$ of studied patients who had low trait anxiety were male, while $(4.00 \%)$ were female.

As regards to marital status, more than two fifth $(40.67 \%)$ of studied patients who had severe trait anxiety were married, while $(2.67 \%)$ were single. On other hands $(12.67 \%)$ of studied patients who had low trait anxiety were married, while (2.67\%) were single.

Regarding level of education, (30.00\%) of studied patients who had severe trait anxiety were illiterate, compared to $(4.00 \%)$ of them were graduated from university. While $(8.67 \%)$ of studied patients who had low trait anxiety were illiterate, compared to $(2.00 \%)$ of them were graduated from university.

Table (9) reveals correlation between studied patient's total knowledge score and The State Trait Anxiety Inventory Scale. It can be seen that, there was a high negative significant correlation $\quad(\mathrm{r}=$ $0.256,-0.318$ respectively) between knowledge and The STAI Scale, $\mathrm{P}$ value $<0.001$.

On other hands, there was a high positive significant correlation $(\mathrm{r}=0.712)$ between The SAI Scale and The TAI Scale, $\mathrm{P}$ value $<0.001$. 
Table (1): Percentage distribution of studied patients according to socio demographic characteristics.

\begin{tabular}{|c|c|c|}
\hline Variables & n (150) & $\%$ \\
\hline $\begin{array}{l}\text { Age (years) } \\
-30-40 \\
-41-50 \\
-51-60\end{array}$ & $\begin{array}{l}23 \\
36 \\
91\end{array}$ & $\begin{array}{l}15.33 \\
24.00 \\
60.67\end{array}$ \\
\hline- Mean \pm SD & $52.56 \pm 12.0$ & \\
\hline $\begin{array}{l}\text { Sex } \\
\text {-Male } \\
\text {-Female } \\
\end{array}$ & $\begin{array}{c}107 \\
43 \\
\end{array}$ & $\begin{array}{r}71.33 \\
28.67 \\
\end{array}$ \\
\hline $\begin{array}{l}\text { Marital status } \\
\text {-Single } \\
\text {-Married } \\
\text {-Divorced } \\
\text {-Widow }\end{array}$ & $\begin{array}{c}10 \\
124 \\
6 \\
10\end{array}$ & $\begin{array}{c}6.67 \\
82.67 \\
4.00 \\
6.67\end{array}$ \\
\hline $\begin{array}{l}\text { Residence } \\
\text {-Urban } \\
\text {-Rural }\end{array}$ & $\begin{array}{c}42 \\
108 \\
\end{array}$ & $\begin{array}{l}28.00 \\
72.00 \\
\end{array}$ \\
\hline $\begin{array}{l}\text { Income level } \\
\text {-Low } \\
\text {-Middle } \\
\text {-High }\end{array}$ & $\begin{array}{l}94 \\
38 \\
18\end{array}$ & $\begin{array}{l}62.67 \\
25.33 \\
12.00\end{array}$ \\
\hline $\begin{array}{l}\text { Education } \\
\text {-Illiterate } \\
\text {-Read and write } \\
\text {-Primary education } \\
\text {-Secondary education } \\
\text {-University }\end{array}$ & $\begin{array}{l}81 \\
31 \\
12 \\
10 \\
16\end{array}$ & $\begin{array}{c}54.00 \\
20.67 \\
8.00 \\
6.67 \\
10.67\end{array}$ \\
\hline $\begin{array}{l}\text { Occupation } \\
\text {-Work } \\
\text {-Not work }\end{array}$ & $\begin{array}{l}54 \\
96\end{array}$ & $\begin{array}{l}36.00 \\
64.00\end{array}$ \\
\hline
\end{tabular}


Table (2): Percentage distribution of studied patients according to current diagnosis and past medical history.

\begin{tabular}{|c|c|c|}
\hline Variables & $\mathbf{n}$ & $\%$ \\
\hline $\begin{array}{l}\text { Admitted from } \\
\text {-Outpatients } \\
\text {-Emergency } \\
\text {-Referred } *\end{array}$ & $\begin{array}{l}56 \\
27 \\
67\end{array}$ & $\begin{array}{l}37.33 \\
18.00 \\
44.67\end{array}$ \\
\hline $\begin{array}{l}\text { Diagnosis } \\
\text {-Esophageal varices and ulcer } \\
\text { Esophageal varices grade I- } \\
\text {-Esophageal varices grade II } \\
\text {-Esophageal varices grade III } \\
\text {-Esophageal stenosis } \\
\text {-Gastric ulcer } \\
\text {-Abdominal pain and distention }\end{array}$ & $\begin{array}{l}43 \\
18 \\
33 \\
18 \\
12 \\
18 \\
8\end{array}$ & $\begin{array}{c}28.67 \\
12.00 \\
22.00 \\
12.00 \\
8.00 \\
12.00 \\
5.33\end{array}$ \\
\hline $\begin{array}{l}\text { Past medical history } \\
\text {-No } \\
\text {-Diabetes mellitus } \\
\text {-Renal diseases } \\
\text {-Hypertension } \\
\text {-Liver and spleen diseases } \\
\text { Pulmonary diseases- } \\
\text {-Cancer } \\
\text { Heart diseases- } \\
\text { Blood diseases- }\end{array}$ & $\begin{array}{c}38 \\
23 \\
5 \\
11 \\
54 \\
5 \\
3 \\
6 \\
5\end{array}$ & $\begin{array}{c}25.33 \\
15.33 \\
3.33 \\
7.33 \\
36.00 \\
3.33 \\
2.00 \\
4.00 \\
3.33\end{array}$ \\
\hline $\begin{array}{l}\text { Family history } \\
\text {-No } \\
\text {-Diabetes mellitus } \\
\text {-Renal diseases } \\
\text {-Liver diseases } \\
\text {-Heart diseases } \\
\text {-Blood diseases }\end{array}$ & $\begin{array}{c}113 \\
16 \\
7 \\
11 \\
1 \\
2\end{array}$ & $\begin{array}{c}75.33 \\
10.67 \\
4.67 \\
7.33 \\
0.67 \\
1.33\end{array}$ \\
\hline $\begin{array}{l}\text { History of hospitalization } \\
\text { Yes- } \\
\text {-No } \\
\text { If yes, for what } \\
\text {-Medical** } \\
\text {-Surgical }\end{array}$ & $\begin{array}{l}68 \\
82\end{array}$ & $\begin{array}{l}45.33 \\
54.67\end{array}$ \\
\hline
\end{tabular}

* From other departments as rural hospital or specialized clinics.

** Mainly liver diseases. 
Table (3): Percentage distribution of studied patients according to history of upper gastrointestinal bleeding.

\begin{tabular}{|c|c|c|}
\hline Variables & $\mathbf{n}$ & $\%$ \\
\hline $\begin{array}{l}\text { Source of information about endoscopy* } \\
\text {-Medical team } \\
\text {-Friends } \\
\text {-Media }\end{array}$ & $\begin{array}{l}73 \\
36 \\
30\end{array}$ & $\begin{array}{l}57.03 \\
28.13 \\
23.44\end{array}$ \\
\hline $\begin{array}{l}\text { History of upper gastrointestinal (GI) bleeding } \\
\text {-Yes } \\
\text {-No }\end{array}$ & $\begin{array}{c}116 \\
34\end{array}$ & $\begin{array}{l}77.33 \\
22.67\end{array}$ \\
\hline $\begin{array}{l}\text { If yes, how many times } \\
\text {-One } \\
\text {-Two } \\
\text {-More }\end{array}$ & $\begin{array}{l}59 \\
26 \\
31\end{array}$ & $\begin{array}{l}50.86 \\
22.41 \\
26.72\end{array}$ \\
\hline $\begin{array}{l}\text { The last attack of bleeding } \\
-<\text { one month } \\
-1-<6 \text { months } \\
-6 \text { months - one year } \\
->\text { one year }\end{array}$ & $\begin{array}{c}49 \\
8 \\
31 \\
28\end{array}$ & $\begin{array}{c}42.24 \\
6.90 \\
26.72 \\
24.14\end{array}$ \\
\hline $\begin{array}{l}\text { There are factors that participate bleeding } \\
\text {-Yes } \\
\text {-No }\end{array}$ & $\begin{array}{l}44 \\
72 \\
\end{array}$ & $\begin{array}{l}37.93 \\
62.07\end{array}$ \\
\hline $\begin{array}{l}\text { If yes, what are these } \\
\text {-Constipation } \\
\text {-Coughing or sneezing } \\
\text {-High blood pressure } \\
\text {-Emotional stress }\end{array}$ & $\begin{array}{c}7 \\
16 \\
9 \\
12\end{array}$ & $\begin{array}{l}15.91 \\
36.36 \\
20.45 \\
27.27\end{array}$ \\
\hline
\end{tabular}

Figure (1): Percentage distribution of studied patients in relation to their total knowledge score regarding upper gastrointestinal endoscopy.

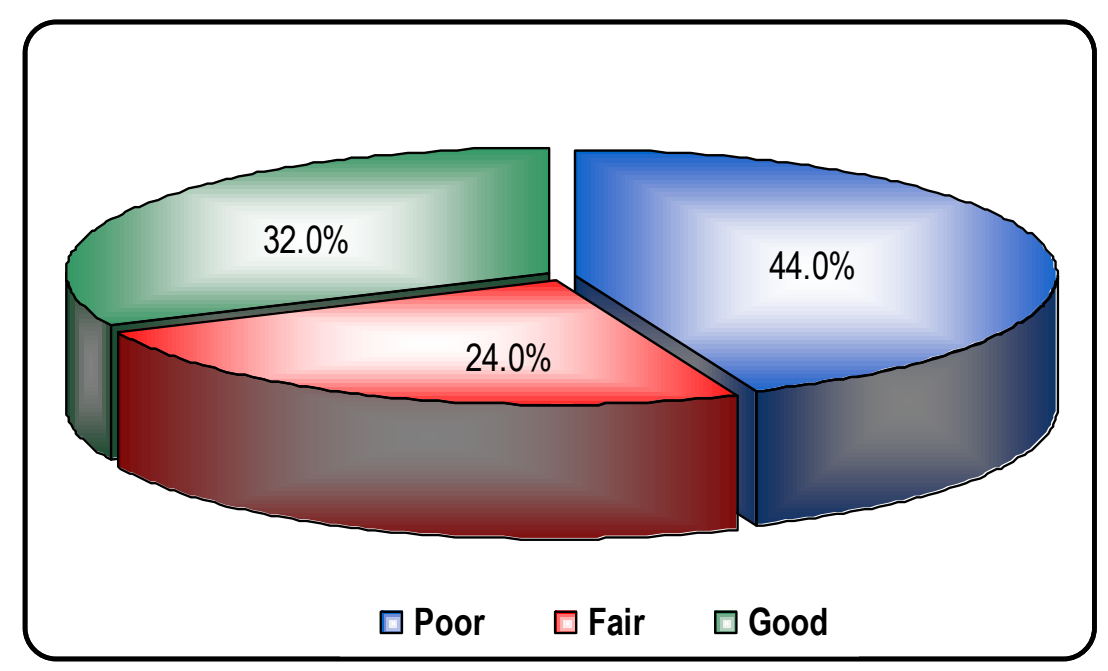


Table (4): Percentage distribution of studied patients in relation to their total Anxiety Inventory Scale pre upper gastrointestinal endoscopy procedure.

\begin{tabular}{|c|c|c|c|c|c|c|c|c|c|c|}
\hline & \multicolumn{9}{|c|}{ Anxiety Inventory Scale } \\
\cline { 2 - 12 } & \multicolumn{2}{|c|}{ Normal } & \multicolumn{2}{|c|}{ Low } & \multicolumn{1}{|c|}{ Moderate } & \multicolumn{2}{c|}{ Severe } & \multicolumn{2}{c|}{ Chi-square } \\
\cline { 2 - 11 } & $\mathbf{n}$ & $\boldsymbol{N}$ & $\mathbf{N}$ & $\boldsymbol{\%}$ & $\mathbf{n}$ & $\boldsymbol{\%}$ & $\mathbf{n}$ & $\%$ & $\mathbf{X}^{\mathbf{2}}$ & P-value \\
\hline -The State & 28 & 18.67 & 15 & 10.00 & 39 & 26.00 & 68 & 45.33 & 40.773 & $<0.001 * *$ \\
\hline -The Trait & 18 & 12.00 & 24 & 16.00 & 31 & 20.67 & 77 & 51.33 & 57.733 & $<0.001 * *$ \\
\hline
\end{tabular}

Table (5): Relation between studied patient's total knowledge score and the Anxiety Inventory Scale.

\begin{tabular}{|c|c|c|c|c|c|c|c|c|c|c|}
\hline \multirow{3}{*}{$\begin{array}{c}\text { Anxiety } \\
\text { Inventory } \\
\text { Scale }\end{array}$} & \multicolumn{10}{|c|}{ Knowledge Score } \\
\hline & \multicolumn{2}{|c|}{ Poor } & \multicolumn{2}{|c|}{ Fair } & \multicolumn{2}{|c|}{ Good } & \multicolumn{2}{|c|}{ Total } & \multicolumn{2}{|c|}{ Chi-square } \\
\hline & $\mathbf{n}$ & $\%$ & $\mathbf{N}$ & $\%$ & $\mathbf{n}$ & $\%$ & $\mathbf{n}$ & $\%$ & $\mathbf{X}^{2}$ & P-value \\
\hline The State & \multirow[b]{2}{*}{5} & \multirow[b]{2}{*}{17.86} & \multirow[b]{2}{*}{9} & \multirow[b]{2}{*}{32.14} & \multirow[b]{2}{*}{14} & \multirow[b]{2}{*}{50.00} & \multicolumn{4}{|c|}{ The State $\left(r=-0.256 / P=0.020^{*}\right)$} \\
\hline -Normal & & & & & & & 28 & 18.67 & & \\
\hline -Low & 7 & 46.67 & 4 & 26.67 & 4 & 26.67 & 15 & 10.00 & 15.466 & $0.017 *$ \\
\hline -Moderate & 14 & 35.90 & 10 & 25.64 & 15 & 38.46 & 39 & 26.00 & & \\
\hline -Severe & 40 & 58.82 & 13 & 19.12 & 15 & \multirow[t]{2}{*}{22.06} & 68 & 45.33 & & \\
\hline The Trait & \multirow[b]{2}{*}{3} & \multirow[b]{2}{*}{16.67} & \multirow[b]{2}{*}{6} & \multirow[b]{2}{*}{33.33} & \multirow[b]{2}{*}{9} & & \multicolumn{4}{|c|}{ The Trait $\quad(r=-0.318 / P<0.001 * *)$} \\
\hline -Normal & & & & & & 50.00 & 18 & 12.00 & & \multirow{4}{*}{$<0.001 * *$} \\
\hline -Low & 9 & 37.50 & 6 & 25.00 & 9 & 37.50 & 24 & 16.00 & 23.510 & \\
\hline -Moderate & 8 & 25.81 & 6 & 19.35 & 17 & 54.84 & 31 & 20.67 & & \\
\hline -Severe & 46 & 59.74 & 18 & 23.38 & 13 & 16.88 & 77 & 51.33 & & \\
\hline
\end{tabular}

Non significant $>0.05$ significant $<0.05 *$ High significant $<0.001 * *$ 
Table (6): Relation between socio demographic data of studied patients and their total knowledge score.

\begin{tabular}{|c|c|c|c|c|c|c|c|c|c|c|}
\hline \multirow{3}{*}{ Variables } & \multicolumn{10}{|c|}{ Knowledge } \\
\hline & \multicolumn{2}{|c|}{ Poor } & \multicolumn{2}{|c|}{ Fair } & \multicolumn{2}{|c|}{ Good } & \multicolumn{2}{|c|}{ Total } & \multicolumn{2}{|c|}{ Tests } \\
\hline & $\mathbf{n}$ & $\%$ & n & $\%$ & $\mathbf{n}$ & $\%$ & $\mathbf{n}$ & $\%$ & $X^{2}$ or $f$ & P-value \\
\hline Age & & & & & & & & & & \\
\hline$-\mathrm{Mean} \pm \mathrm{SD}$ & \multicolumn{2}{|c|}{$47.32 \pm 11.15$} & \multicolumn{2}{|c|}{$49.67 \pm 10.62$} & \multicolumn{2}{|c|}{$52.18 \pm 8.35$} & & & 3.695 & $0.027 *$ \\
\hline Sex & & & & & & & & & & \\
\hline -Male & 47 & 31.33 & 29 & 19.33 & 31 & 20.67 & 107 & 71.33 & 2.567 & 0.277 \\
\hline -Female & 19 & 12.67 & 7 & 4.67 & 17 & 11.33 & 43 & 28.67 & & \\
\hline Marital status & & & & & & & & & & \\
\hline -Single & 6 & 4.00 & 1 & 0.67 & 3 & 2.00 & 10 & 6.67 & 23.030 & $<0.001 * *$ \\
\hline -Married & 49 & 32.67 & 35 & 23.33 & 40 & 26.67 & 124 & 82.67 & & \\
\hline -Divorced & 1 & 0.67 & 0 & 0.00 & 5 & 3.33 & 6 & 4.00 & & \\
\hline -Widow & 10 & 6.67 & 0 & 0.00 & 0 & 0.00 & 10 & 6.67 & & \\
\hline Residence & & & & & & & & & & \\
\hline -Urban & 14 & 9.33 & 12 & 8.00 & 16 & 10.67 & 42 & 28.00 & 2.694 & 0.260 \\
\hline -Rural & 52 & 34.67 & 24 & 16.00 & 32 & 21.33 & 108 & 72.00 & & \\
\hline Education & & & & & & & & & & \\
\hline -Illiterate & 38 & 25.33 & 23 & 15.33 & 20 & 13.33 & 81 & 54.00 & 23.205 & $0.003 *$ \\
\hline -Readand write & 12 & 8.00 & 9 & 6.00 & 10 & 6.67 & 31 & 20.67 & & \\
\hline -Primary edu & 9 & 6.00 & 2 & 1.33 & 1 & 0.67 & 12 & 8.00 & & \\
\hline -Secondary edu & 4 & 2.67 & 1 & 0.67 & 5 & 3.33 & 10 & 6.67 & & \\
\hline -University & 3 & 2.00 & 1 & 0.67 & 12 & 8.00 & 16 & 10.67 & & \\
\hline Income level & 50 & 3333 & 23 & 1533 & & & & & 23745 & $<0001 * *$ \\
\hline -LOW & 50 & $\begin{array}{r}33.33 \\
0.67\end{array}$ & 23 & 15.35 & 21 & $\begin{array}{l}14.00 \\
8.67\end{array}$ & $\begin{array}{l}94 \\
20\end{array}$ & $\begin{array}{l}62.67 \\
2523\end{array}$ & 23.745 & $<0.001 * *$ \\
\hline -Middle & 13 & 8.67 & 12 & 8.00 & 13 & 8.67 & 38 & 25.33 & & \\
\hline -High & 3 & 2.00 & 1 & 0.67 & 14 & 9.33 & 18 & 12.00 & & \\
\hline Occupation & & & & & & & & & & \\
\hline -Work & 8 & 5.33 & 12 & 8.00 & 34 & 22.67 & 54 & 36.00 & 41.723 & $<0.001 * *$ \\
\hline -Not work & 58 & 38.67 & 24 & 16.00 & 14 & 9.33 & 96 & 64.00 & & \\
\hline
\end{tabular}

Non significant $>0.05$ significant $<0.05 *$ High significant $<0.001$ 
Table (7): Relation between socio demographic data of studied patients and The State Anxiety Inventory Scale.

\begin{tabular}{|c|c|c|c|c|c|c|c|c|c|c|c|c|}
\hline \multirow{3}{*}{ Variables } & \multicolumn{12}{|c|}{ The State Anxiety Inventory scale } \\
\hline & \multicolumn{2}{|c|}{ Normal } & \multicolumn{2}{|c|}{ Low } & \multicolumn{2}{|c|}{$\begin{array}{l}\text { Moderat } \\
\text { e }\end{array}$} & \multicolumn{2}{|c|}{ Severe } & \multicolumn{2}{|c|}{ Total } & \multicolumn{2}{|c|}{ Tests } \\
\hline & n & $\%$ & $\mathbf{n}$ & $\%$ & & & $\mathbf{n}$ & $\%$ & n & $\%$ & $X^{2}$ or $f$ & P-value \\
\hline $\begin{array}{l}\text { Age (years) } \\
-M e a n \pm S D\end{array}$ & 56.29 & \pm 11.48 & 54.2 & 12.19 & 50.1 & $=10.24$ & 51.6 & 8.51 & & & 2.373 & 0.073 \\
\hline $\begin{array}{l}\text { Sex } \\
\text {-Male } \\
\text {-Female } \\
\end{array}$ & $\begin{array}{c}24 \\
4 \\
\end{array}$ & $\begin{array}{c}16.00 \\
2.67 \\
\end{array}$ & $\begin{array}{c}11 \\
4\end{array}$ & $\begin{array}{l}7.33 \\
2.67\end{array}$ & $\begin{array}{l}29 \\
10 \\
\end{array}$ & $\begin{array}{r}19.33 \\
6.67 \\
\end{array}$ & $\begin{array}{l}43 \\
25\end{array}$ & $\begin{array}{l}28.67 \\
16.67\end{array}$ & $\begin{array}{r}107 \\
43 \\
\end{array}$ & $\begin{array}{l}71.33 \\
28.67\end{array}$ & 5.216 & 0.157 \\
\hline $\begin{array}{l}\text { Marital status } \\
\text {-Single } \\
\text {-Married } \\
\text {-Divorced } \\
\text {-Widow }\end{array}$ & $\begin{array}{c}1 \\
24 \\
3 \\
0\end{array}$ & $\begin{array}{c}0.67 \\
16.00 \\
2.00 \\
0.00\end{array}$ & $\begin{array}{c}3 \\
12 \\
0 \\
0\end{array}$ & $\begin{array}{l}2.00 \\
8.00 \\
0.00 \\
0.00\end{array}$ & $\begin{array}{c}2 \\
33 \\
2 \\
2\end{array}$ & $\begin{array}{c}1.33 \\
22.00 \\
1.33 \\
1.33\end{array}$ & $\begin{array}{c}4 \\
55 \\
1 \\
8\end{array}$ & $\begin{array}{c}2.67 \\
36.67 \\
0.67 \\
5.33\end{array}$ & $\begin{array}{c}10 \\
124 \\
6 \\
10\end{array}$ & $\begin{array}{c}6.67 \\
82.67 \\
4.00 \\
6.67\end{array}$ & 15.316 & 0.083 \\
\hline $\begin{array}{l}\text { Residence } \\
\text {-Urban } \\
\text {-Rural }\end{array}$ & $\begin{array}{c}4 \\
24\end{array}$ & $\begin{array}{c}2.67 \\
16.00\end{array}$ & $\begin{array}{l}6 \\
9\end{array}$ & $\begin{array}{l}4.00 \\
6.00\end{array}$ & $\begin{array}{l}14 \\
25\end{array}$ & $\begin{array}{c}9.33 \\
16.67\end{array}$ & $\begin{array}{l}18 \\
50\end{array}$ & $\begin{array}{l}12.00 \\
33.33\end{array}$ & $\begin{array}{c}42 \\
108\end{array}$ & $\begin{array}{l}28.00 \\
72.00\end{array}$ & 4.969 & 0.174 \\
\hline $\begin{array}{l}\text { Education level } \\
\text {-Illiterate } \\
\text {-Read and write } \\
\text {-Primary edu } \\
\text {-Secondary edu } \\
\text {-University }\end{array}$ & $\begin{array}{l}14 \\
3 \\
0 \\
4 \\
7\end{array}$ & $\begin{array}{l}9.33 \\
2.00 \\
0.00 \\
2.67 \\
4.67\end{array}$ & $\begin{array}{l}9 \\
3 \\
0 \\
1 \\
2\end{array}$ & $\begin{array}{l}6.00 \\
2.00 \\
0.00 \\
0.67 \\
1.33\end{array}$ & $\begin{array}{c}23 \\
9 \\
1 \\
3 \\
3\end{array}$ & $\begin{array}{c}15.33 \\
6.00 \\
0.67 \\
2.00 \\
2.00\end{array}$ & $\begin{array}{c}35 \\
16 \\
11 \\
2 \\
4\end{array}$ & $\begin{array}{r}23.33 \\
10.67 \\
7.33 \\
1.33 \\
2.67\end{array}$ & $\begin{array}{l}81 \\
31 \\
12 \\
10 \\
16\end{array}$ & \begin{tabular}{|c|}
54.00 \\
20.67 \\
8.00 \\
6.67 \\
10.67
\end{tabular} & 23.921 & $0.021^{*}$ \\
\hline $\begin{array}{l}\text { Income level } \\
\text {-Low } \\
\text {-Middle } \\
\text {-High }\end{array}$ & $\begin{array}{c}14 \\
7 \\
7\end{array}$ & $\begin{array}{l}9.33 \\
4.67 \\
4.67\end{array}$ & $\begin{array}{l}7 \\
6 \\
2\end{array}$ & $\begin{array}{l}4.67 \\
4.00 \\
1.33\end{array}$ & $\begin{array}{c}24 \\
13 \\
2\end{array}$ & $\begin{array}{l}16.00 \\
8.67 \\
1.33\end{array}$ & $\begin{array}{c}49 \\
12 \\
7\end{array}$ & $\begin{array}{r}32.67 \\
8.00 \\
4.67\end{array}$ & $\begin{array}{l}94 \\
38 \\
18\end{array}$ & $\begin{array}{l}62.67 \\
25.33 \\
12.00\end{array}$ & 11.806 & 0.066 \\
\hline $\begin{array}{l}\text { Occupation } \\
\text {-Work } \\
\text {-Not work }\end{array}$ & $\begin{array}{l}15 \\
13\end{array}$ & $\begin{array}{c}10.00 \\
8.67\end{array}$ & $\begin{array}{l}6 \\
9\end{array}$ & $\begin{array}{l}4.00 \\
6.00\end{array}$ & $\begin{array}{l}17 \\
22\end{array}$ & $\begin{array}{l}11.33 \\
14.67\end{array}$ & $\begin{array}{l}16 \\
52\end{array}$ & $\begin{array}{l}10.67 \\
34.67\end{array}$ & $\begin{array}{l}54 \\
96\end{array}$ & $\begin{array}{l}36.00 \\
64.00\end{array}$ & 9.421 & $0.024 *$ \\
\hline
\end{tabular}

Non significant $>0.05$ significant $<0.05 *$ High significant $<0.001 * *$ 
Table (8): Relation between socio demographic data of studied patients and The Trait Anxiety Inventory Scale.

\begin{tabular}{|c|c|c|c|c|c|c|c|c|c|c|c|c|}
\hline \multirow{3}{*}{ Variables } & \multicolumn{10}{|c|}{ The Trait Anxiety Inventory scale } & & \\
\hline & \multicolumn{2}{|c|}{ Normal } & \multicolumn{2}{|c|}{ Low } & \multicolumn{2}{|c|}{ Moderate } & \multicolumn{2}{|c|}{ Severe } & \multicolumn{2}{|c|}{ Total } & \multicolumn{2}{|c|}{ Tests } \\
\hline & $\mathbf{n}$ & $\%$ & $\mathbf{N}$ & $\%$ & $\mathrm{n}$ & $\%$ & $\bar{n}$ & $\%$ & $\mathbf{n}$ & $\%$ & $\mathbf{X}^{2}$ or $\mathbf{f}$ & P-value \\
\hline $\begin{array}{l}\text { Age (years) } \\
\text {-Mean } \pm \text { SD }\end{array}$ & \multicolumn{2}{|c|}{$53.66 \pm 12.82$} & \multicolumn{2}{|c|}{$55.92 \pm 8.7$} & \multicolumn{2}{|c|}{$52.73 \pm 12.54$} & \multicolumn{2}{|c|}{$51.62 \pm 11.58$} & & & 0.887 & 0.450 \\
\hline $\begin{array}{l}\text { Sex } \\
\text {-Male } \\
\text {-Female } \\
\end{array}$ & $\begin{array}{c}14 \\
4 \\
\end{array}$ & $\begin{array}{l}9.33 \\
2.67 \\
\end{array}$ & $\begin{array}{c}18 \\
6 \\
\end{array}$ & $\begin{array}{c}12.00 \\
4.00 \\
\end{array}$ & $\begin{array}{c}22 \\
9 \\
\end{array}$ & $\begin{array}{c}14.67 \\
6.00 \\
\end{array}$ & $\begin{array}{l}53 \\
24 \\
\end{array}$ & $\begin{array}{l}35.33 \\
16.00\end{array}$ & $\begin{array}{c}107 \\
43 \\
\end{array}$ & $\begin{array}{l}71.33 \\
28.67 \\
\end{array}$ & 0.761 & 0.859 \\
\hline $\begin{array}{l}\text { Marital } \\
\text { status } \\
\text {-Single } \\
\text {-Married } \\
\text {-Divorced } \\
\text {-Widow }\end{array}$ & $\begin{array}{c}1 \\
16 \\
1 \\
0 \\
\end{array}$ & $\begin{array}{c}0.67 \\
10.67 \\
0.67 \\
0.00 \\
\end{array}$ & $\begin{array}{c}4 \\
19 \\
0 \\
1 \\
\end{array}$ & $\begin{array}{c}2.67 \\
12.67 \\
0.00 \\
0.67 \\
\end{array}$ & $\begin{array}{c}1 \\
28 \\
1 \\
1 \\
\end{array}$ & $\begin{array}{c}0.67 \\
18.67 \\
0.67 \\
0.67 \\
\end{array}$ & $\begin{array}{c}4 \\
61 \\
4 \\
8\end{array}$ & $\begin{array}{c}2.67 \\
40.67 \\
2.67 \\
5.33\end{array}$ & $\begin{array}{c}10 \\
124 \\
6 \\
10\end{array}$ & $\begin{array}{c}6.67 \\
82.67 \\
4.00 \\
6.67\end{array}$ & 9.851 & 0.363 \\
\hline $\begin{array}{l}\text { Residence } \\
\text {-Urban } \\
\text {-Rural }\end{array}$ & $\begin{array}{c}7 \\
11\end{array}$ & $\begin{array}{l}4.67 \\
7.33\end{array}$ & $\begin{array}{l}11 \\
13\end{array}$ & $\begin{array}{l}7.33 \\
8.67\end{array}$ & $\begin{array}{l}10 \\
21\end{array}$ & $\begin{array}{c}6.67 \\
14.00\end{array}$ & $\begin{array}{l}14 \\
63\end{array}$ & $\begin{array}{c}9.33 \\
42.00\end{array}$ & $\begin{array}{c}42 \\
108\end{array}$ & $\begin{array}{l}28.00 \\
72.00\end{array}$ & 8.805 & $0.032 *$ \\
\hline $\begin{array}{l}\text { Education } \\
\text {-Illiterate } \\
\text {-Read and } \\
\text { write } \\
\text {-Primary } \\
\text { education } \\
\text {-Secondary } \\
\text { education } \\
\text {-University }\end{array}$ & $\begin{array}{l}4 \\
3 \\
\end{array}$ & $\begin{array}{l}0.67 \\
2.67 \\
2.00 \\
\end{array}$ & $\begin{array}{c}13 \\
7\end{array}$ & $\begin{array}{l}8.67 \\
4.67 \\
0.00 \\
0.67 \\
2.00 \\
\end{array}$ & $\begin{array}{c}15 \\
6\end{array}$ & $\begin{array}{l}10.00 \\
4.00 \\
2.00 \\
2.00 \\
2.67 \\
\end{array}$ & 45 & $\begin{array}{c}30.00 \\
10.67 \\
5.33 \\
1.33 \\
4.00 \\
\end{array}$ & $\begin{array}{l}81 \\
31\end{array}$ & $\begin{array}{c}54.00 \\
20.67 \\
8.00 \\
\\
6.67 \\
10.67 \\
\end{array}$ & 15.658 & 0.207 \\
\hline $\begin{array}{l}\text { Income level } \\
\text {-Low } \\
\text {-Middle } \\
\text {-High } \\
\end{array}$ & $\begin{array}{l}7 \\
6 \\
5 \\
\end{array}$ & $\begin{array}{l}4.67 \\
4.00 \\
3.33 \\
\end{array}$ & $\begin{array}{c}16 \\
6 \\
2 \\
\end{array}$ & $\begin{array}{c}10.67 \\
4.00 \\
1.33 \\
\end{array}$ & $\begin{array}{c}18 \\
10 \\
3 \\
\end{array}$ & $\begin{array}{c}12.00 \\
6.67 \\
2.00 \\
\end{array}$ & $\begin{array}{c}53 \\
16 \\
8 \\
\end{array}$ & $\begin{array}{c}35.33 \\
10.67 \\
5.33 \\
\end{array}$ & $\begin{array}{l}94 \\
38 \\
18 \\
\end{array}$ & $\begin{array}{l}62.67 \\
25.33 \\
12.00 \\
\end{array}$ & 8.239 & 0.221 \\
\hline $\begin{array}{l}\text { Occupation } \\
\text {-Work } \\
\text {-Not work }\end{array}$ & $\begin{array}{c}13 \\
5\end{array}$ & $\begin{array}{r}8.67 \\
3.33\end{array}$ & $\begin{array}{c}9 \\
15\end{array}$ & $\begin{array}{c}6.00 \\
10.00\end{array}$ & $\begin{array}{l}15 \\
16\end{array}$ & $\begin{array}{l}10.00 \\
10.67\end{array}$ & $\begin{array}{l}17 \\
60\end{array}$ & $\begin{array}{l}11.33 \\
40.00\end{array}$ & $\begin{array}{l}54 \\
96\end{array}$ & $\begin{array}{l}36.00 \\
64.00\end{array}$ & 18.816 & $<0.001 * *$ \\
\hline
\end{tabular}

Non significant $>0.05$ significant $<0.05 *$ High significant $<0.001 *$

Table (9): Correlation between studied patient's total knowledge score and The State Trait Anxiety Inventory Scale.

\begin{tabular}{|c|c|c|c|c|}
\hline \multirow{2}{*}{ Variables } & \multicolumn{2}{|c|}{ Knowledge } & \multicolumn{2}{|c|}{ The STAI Scale } \\
\hline & $\mathbf{R}$ & P-value & $\mathbf{r}$ & P-value \\
\hline $\begin{array}{l}\text { The State Anxiety Inventory (SAI) } \\
\text { Scale }\end{array}$ & -0.256 & $0.020^{*}$ & & \\
\hline $\begin{array}{l}\text { The Trait Anxiety Inventory (TAI) } \\
\text { Scale }\end{array}$ & -0.318 & $<0.001 * *$ & 0.712 & $<0.001 * *$ \\
\hline
\end{tabular}




\section{Discussion:}

The upper gastrointestinal endoscopic procedure anxiety was often associated with the fear of experiencing pain and discomfort during endoscopy, decreases patient cooperation and tolerance with procedure $^{(32)}$. Therefore, control of discomfort and pain before the endoscopy was considered to be a high priority by patients. The best intervention to reduce anxiety is to inform patient with adequate knowledge about the procedure ${ }^{(33)}$. For these reasons, the current study aimed to assess knowledge and anxiety level of patients undergoing upper GI endoscopy at Tanta University Hospital.

\section{Regarding socio demographic characteristic:}

Less than two thirds of studied patients who undergoing upper GI endoscopy was in age group ranged from 51 to 60 years. This could be explained that this age represent last period of working age population which exposed to unhealthy life style such as stressful life events, misuse of medications as analgesics that effect on gastro intestinal tract, and increased consumption of fast food, spicy and high fat diet. This finding was inagreement with Ruhl and Everhart (2016) $^{(34)}$ in a study to "Assess indications and outcomes needs of patients undergoing gastrointestinal endoscopy". They stated that the majority of patients undergoing upper GI endoscopy belonged to the age group 5059 years.

On other hand, this finding was contradicted by Lee et al (2012) $)^{(35)}$ in a study of " The effect of preparatory education program on discomfort during upper gastrointestinal endoscopy "and EI said (2016) ${ }^{(36)}$ in a study entitled "Identify effectiveness of structured teaching program on knowledge, anxiety state and tolerance for patients with GI endoscopy", they mentioned that, most of patients undergoing upper GI endoscopy were belonged to the age 41 years.

Moreover, in the current study it was found that more than two thirds of studied patients were male. This could be explained that male patients exposed to stressful life, heavy smoking and increasing consumption of caffeine more than female patients. This finding was highly supported with study done by Seda et al (2011) $)^{(37)}$ in a study to determine "Effect of providing information to the patient about upper gastrointestinal endoscopy on the patient's perception, compliance and anxiety level", they found that the majority of studied patients who undergoing upper GI endoscopy were male. 
Also this finding was inconsistent with Smitha and Sugirtha $(\mathbf{2 0 1 5})^{(38)}$ in a study to "Assess effectiveness of structured teaching programme on knowledge and anxiety of patients undergoing endoscopy", they stated that majority of patients were predominantly male. On the other hand, this result was not accordance with study done by Feyzullah et al (2010) $)^{(39)}$ in a study entitled "Assessment of anxiety levels in patients during elective upper gastrointestinal endoscopy and colonoscopy "to assess anxiety levels in patients during elective upper gastro intestinal endoscopy, they reported that upper GI endoscopy more common in women than in men.

Regarding marital status, the current study revealed that more than three quarters of studied patients were married, this finding was in harmony with Mohammed(2016) $)^{(40)}$ in a study to determine "Effectiveness of structured teaching program on knowledge, anxiety state and tolerance for patients with gastrointestinal endoscopy", who recorded that nearly two thirds of studied patients were married. Also, this finding was in line with Abdulla (2010) ${ }^{(41)}$ who reported that the majority of patients in a study entitled; "Assessment of knowledge and perception of the patient with upper gastrointestinal disorders" were married.

As regards to residence, the current study showed that more than two thirds of studied patients had lived in rural. It may be due to people lived in rural area usually ignore any pain suffers and neglect routine checkup, also they doesn't find big hospitals provide intensive care. This finding inagreement with Prabhuswami et al (2016) ${ }^{(42)}$ in a study to "Assess the knowledge, attitude and pre procedure anxiety level of patients undergoing upper gastrointestinal endoscopy", they stated that the majority of studied patients came from rural area. Also, this result was supported by Kennedy et al (2006) ${ }^{(43)}$ and Vliet et al (2004) ${ }^{(44)}$, they illustrated that rural patients with lowest socioeconomic status were high risk for upper GI diseases.

Concerning income level, in the current study it was found that less than two thirds of studied patients consider low income level, more than half were illiterate and nearly two thirds of them had no work. This may be interpreted that poor income and low educational levels had negative effects on health, and these events may affect knowledge and anxiety level among patients. This finding was supported by Quick (2006) ${ }^{(45)}$ who revealed that 
illiterate and low income level patient did not seek the hospital or clinics except in cases of necessity only, and for this reason the medical problem became severe.

In addition, this finding was consistent with Winslow (2004) ${ }^{(46)}$ and Mohamed et al (2014) $)^{(47)}$, they mentioned that; the majority of studied patient who undergoing upper GI endoscopy were illiterate and had low income level. $n$ the other hand, this finding was contradicted by study done with Previti et al $\mathbf{( 2 0 1 6 )}^{(48)}$ in a study entitled "Identifying people at risk for anxiety in patients undergoing endoscopic procedures", they revealed that more than two thirds of studied patients who undergoing upper GI endoscopy had high education level.

\section{Knowledge about upper gastrointestinal endoscopy:}

Upper gastrointestinal endoscopy procedure is a difficult, stressful, anxiety associated, unpleasant diagnostic and therapeutic method (49-51). Providing adequate information before upper GI endoscopy lead to decrease anxiety, fear and worry of the patients during the procedure $^{(52-56)}$.

For that, an effective health teaching guidelines should be given, depending on the patient's individual needs and the patient should be assisted in order to establish good compliance throughout the procedure $^{(57-61)}$.

Regarding patient's knowledge before upper GI endoscopy; it can be seen from current study that less than half of studied patients had unsatisfied knowledge score regarding upper GI endoscopy, while less than one third of them had good knowledge score .

Additionally, these results suggested that providing verbal information to the patients is effective and essential in increasing knowledge about the nature and duration of the upper GI endoscopy, helping them to feel secure and reducing their anxiety associated with the result of the procedure. This finding was contradicted by Prabhuswami et al $(\mathbf{2 0 1 6})^{(42)}$, they mentioned that the majority of studied patients who undergoing upper GI endoscopy had good knowledge.

In order to provide sufficient information to studied patients before undergoing upper GI endoscopy, the information should cover different issues such as: Indication, types, possible occurrence of discomfort or side effect, physical and psychological preparation before endoscopy ${ }^{(62-64)}$.Also, these findings were inagreement with study done by Thomas 
and Sugirtha $(\mathbf{2 0 1 5})^{(38)}$, they illustrated that good preparation by providing adequate knowledge to patient before diagnostic GI endoscopy enhance patients understanding and awareness about this procedure.

\section{III.Regarding upper gastrointestinal endoscopy and anxiety score.}

The finding of this study showed that less than half of studied patients had severe state anxiety, while more than half had severe trait anxiety. These findings may due to lack of awareness about the upper GI endoscopy procedure, fear of discomfort or pain in procedure and/or its results. These findings were insimilar with study done by Jones et al(2004) ${ }^{(65)}$ to determine "Relation between patient's anxiety and elective upper gastro endoscopy", they reported that the anxiety level was increased before the endoscopy procedure.

Also, these findings were inline with Brandt (2001) ${ }^{(66)}$ in a study entitled "Patients' attitudes and apprehensions about endoscopy", who demonstrated that patients scheduled for endoscopy procedure were usually anxious from the procedure. On other hand, these findings were contradicted by Trevisani et al $\mathbf{( 2 0 1 6 )}^{(67)}$ and Essink-Bot et al (2016) ${ }^{(68)}$, they revealed that no correlation between upper GI endoscopy and knowledge and pre procedural anxiety.

\section{Regarding knowledge and anxiety score in upper gastrointestinal endoscopy.}

A statistical significant difference was observed among studied patients in relation to their knowledge and The STAI Scale due to structured information might decrease patient anxiety and improve general health. This result was supported with the study conducted by Ajee (2002) ${ }^{(69)}$ in a study to assess "The effectiveness of structured teaching on anxiety and physiological responses of adult patients undergoing endoscopy procedure" and Aabakken (2010) $)^{(70)}$ in a study entitled "Development and evaluation of written patient information for endoscopic procedure", these studies emphasized that structured information decrease anxiety and improve patient's tolerance level.

In addition, similar results were found by Kiyohara et al(2004) ${ }^{(71)}$ in a study to assess "Anxiety in preoperative anesthetic endoscopy procedure", this study revealed that increase in patient's knowledge regarding upper GI endoscopy procedure reduced their anxiety level.

Moreover, it can be seen that nearly half of studied patients had good knowledge and normal STAI Scale, while more than half 


\section{Tanta Scientific Nursing Journal}

had poor knowledge and severe STAI Scale. This means that adequate information and increase awareness among patients pre upper GI endoscopy procedure associated with reduce the anxiety level. This finding was in agreement with study done by Rohde (2011) $^{(72)}$ in a study entitled "Anxiety before gastrointestinal endoscopy is a significant problem?", who reported that more than half of studied patients who experienced severe anxiety before GI endoscopy had poor knowledge regarding the procedure.

Additionally, this finding was in line with the study done by Peter et al (2008) ${ }^{(73)}$ in a study "The fundamental of upper gastrointestinal endoscopy", they showed that a combination of information and training prior to upper GI endoscopy procedure is an effective means for reducing anxiety.

\section{Socio demographic characteristics and knowledge score regarding upper gastrointestinal endoscopy:}

It was found that a high statistical significant difference between knowledge in relation to sex, marital status, socioeconomic status, education level, income level and occupation. It may be due to more than half of studied patients had low level of education, no occupation and poor socioeconomic status which reflect on acquisition of knowledge. However, those patients had the confidence in themselves and cognitive maturity to get the information that they need through medical teams, reading articles and discussion with others. This result was inconsistent with Mulcahy et al (2010) $^{(74)}$, they illustrated that a study to identify "Factors associated with tolerance and discomfort with unsedated diagnostic gastroscopy", they demonstrated that besides anxiety, the age, education level and gender also influenced significantly the tolerance of studied patients to upper GI endoscopy.

On other hand, this result was contradicted by Hashimot (2002) ${ }^{(75)}$ in a study entitled "Safety and efficacy of glucagon as a pre medication for upper gastro intestinal endoscopy", who pointed that there was no significant difference in relation to sex or age with tolerance to endoscopy.

Also, Lee et al (2004) $)^{(76)}$ in a study entitled "Can visual distraction decrease the dose of patient controlled sedation required during endoscopy" and Campo(2015) ${ }^{(77)}$ in a study entitled "Identification of factors that influence tolerance of upper gastrointestinal endoscopy", they reported that no significant difference in relation to 
patient's age, gender and education level related to upper GI endoscopy.

\section{Socio demographic characteristics} and the Anxiety Inventory Scale regarding upper gastrointestinal endoscopy:

The current study revealed that there was a high statistical significant difference found between The STAI Scale in relation to sex, in fact nearly two thirds of studied patients who had severe STAI were male, while nearly one third of them were female. It may be due to no equality number in both gender of studied sample in this study.

These findings contradicted by Trevisani et al (2002) $)^{(67)}$, Muzzarelli et al $\mathbf{( 2 0 0 6 )}^{(78)}$ and Hakan et al (2012) ${ }^{(79)}$, they identified that anxiety prior to the upper GI endoscopy had been reported to be more severe in women due to fear of not waking up after the procedure than in men.

\section{Correlation between studied} patient's total knowledge score, The State and Trait Anxiety Scale.

The present study demonstrated that there was a negative significant correlation between knowledge and The STAI Scale. It may be due to the recommendation of upper GI endoscopy procedure, increases the patient's apprehension and anxiety that is due to lack of knowledge of the patient about endoscopy, also awaiting the result of endoscopy. Moreover, most of studied patients were illiterate and consider low socioeconomic standard, so financial resource decreased, which lead to increase level of stress and anxiety.

On other hands, there was a positive significant correlation between The SAI and TAI. This means the internal personality, generally feel and trait in life of studied patients had positive effect on them in different situations as immediately before the procedure ${ }^{(80)}$. This finding was consistent with study done by Trevisani et al $\mathbf{( 2 0 0 2}^{(67)}$, they found no statistical significant difference between The SAI and TAI in studied patients who undergoing upper GI endoscopy.

\section{Conclusion and recommendations:}

\section{Conclusion}

Based on the findings of the present study, it can be concluded that:

- There was a negative statistical significant relationship existed between knowledge and the anxiety scale of studied patients, the results revealed that studied patients with good knowledge score appeared in normal anxiety scale, while studied patients who had poor knowledge appeared in severe anxiety scale. 
- Moreover, a positive statistical significant relationship was observed between SAI and TAI scales. In the present study, less than half of studied patients had severe SAI Scale, while more than half had severe TAI Scale.

- The study also revealed that, there were certain factors that influence the knowledge and The STAI Scale of studied patients as sex, marital status, socioeconomic status, income level and occupation.

- Finally, overall findings revealed that good preparation by providing information to the patient before the procedure, decrease their level of anxiety and improve tolerance level.

\section{Recommendations}

Based upon the findings of this study, the following recommendations are derived and suggested:

\section{Recommendation for patients:}

- Counseling should be provided for all patients who are undergoing upper GI endoscopy that helps in preparation of them and reducing anxiety level.

\section{Recommendation for clinical} practice:

- Assessment of patient's knowledge towards upper GI endoscopy must be done upon patient admission by nurses.

- Assessment of patient's anxiety towards upper GI endoscopy must be done in the initial data collection and be documented in patients file by nurses using.

\section{Recommendation for administration:}

- Written policies and guide lines should be available regarding increasing knowledge and declining anxiety for patients undergoing upper GI endoscopy.

- Provision of colored booklet regarding physical and psychological preparation before upper GI procedure.

- Multidisciplinary team should be available to provide individualized information for each patient.

\section{Recommendation for further research} studies:

- Replication of the study on a larger sample in different hospitals and multiple geographical areas, to confirm the result of the study.

- Study is needed for nursing staff to evaluate the effect of nurse's knowledge regarding upper GI endoscopy on patient's outcome. 


\section{References:}

1. Van M, Grypdonck M and Zuuren F. Patients for gastrointestinal endoscopy: The influence of information in medical situations, Patient Education and Counseling J.2004; 52(1):23-30.

2. Hafez A, Hassan E and Abou Rayah F. Endoscopy department of experimental and clinical surgery, Medical research institute in Egypt, International Journal of Nursing. 2010; 4(4):1-5.

3. Hiremath P, Mohite V and Pawar S. A study to assess the knowledge, attitude and pre procedure anxiety level of patient undergoing upper gastrointestinal endoscopy, The American Journal of Gastroenterology.2016; 5(6):242-9.

4. Registration department of gastrointestinal center, Tanta University Hospital 2017.

5. Rudin D. Frequently overlooked and rarely listened to: Music therapy in gastrointestinal endoscopic procedures, World J Gastroenterol. 2007; 13(33): 4518- 33.

6. Robinson J, Moreau D and Mcann J. Hand Book of Medical Surgical Nursing, Pennsylvania: Springhouse.2002; 1(3): 303-10.
7. Rex D and Khalfan H. Sedation and the technical performance of colonoscopy: Gastrointestinal endoscopy clinics of North America, Journal of Clinical Nursing. 2005; 15:661-72.

8. Van L, Rossum $G$ and Oijen M. Anxiety, depression and psychotropic medication use in patients with persistent upper gastrointestinal symptoms, Aliment Pharma TherJ. 2005; 21:1001-6.

9. Oxelmark L, Nordström G, Sjöqvist U and Löfberg R. Anxiety, functional health status, and coping ability in patients with ulcerative colitis who are undergoing colonoscopy surveillance, Inflamm Bowel Dis J. 2004; 10: 612-7.

10. Gomez M and Llach J. Role of nurses in gastrointestinal endoscopy, Gastroenterol Hepatol J. 2009; 32(1):44-7.

11. Salvetto M. The nurse's role in endoscopic ultra sonograph, Minerva Med J.2007;98(1):269-75.

12. Koloski N and Boyce P. Epidemiology and health care seeking in the functional GI disorders: A population based study, Gastroenterol J. 2009; 97 : 2290-9.

13. Locke G, Weaver A and Melton L. Psychosocial factors are linked to 
functional gastrointestinal disorders: Case control study, Gastroenterol J.2004;99: 350-7.

14. Pajala M, Heikkinen M and Hintikka J. Mental distress in patients with functional or organic dyspepsia, Aliment Pharmacol Ther J. 2005; 21: 277-81.

15. El-Hassan H, McKeown K and Muller A. Music reduces anxiety levels in patients attending for endoscopy, Aliment Pharmacol Ther J. 2009;30(7):718-24.

16. Addolorato G, Mirijello A and D'AngeloC. State and trait anxiety and depression in patients affected by gastrointestinal diseases: Psychometric evaluation of 1641 patients referred to an internal medicine outpatient setting, Int J Clin Pract. 2008; 62: 1063-9.

17. Tina $D$ and Steven P. An evaluation of a teaching intervention to improve the practice, Journal of Clinical Nursing. 2006; 10(5):682- 96.

18. Pehlivan S, Ovayolu N and Koruk M. Effect of providing information to patient about upper gastrointestinal endoscopy on the patient's perception, compliance and anxiety level associated with the procedure, Journal of Clinical Nursing. 2011; 22(1):10-27.

19. Alonso J, Angermeyer M, Bernert S,
Bruffaerts R and Brugha T. Study of the epidemiology of mental disorders project. Act Psychiatr Scand Suppl J. 2004; 21:7-9.

20. Annette D and Jean A. Medical Surgical Nursing, Assessment and management of endoscopy, Brown and Edwards Comp, Australia. 2007;3:189206.

21. Poley J, Steyerberg E and Kuipers E. Outcome and prognostic value of early upper endoscopy, Gastrointest Endosc J. 2005; 60(1): 372-7.

22. Berzin $\mathrm{T}$, Blanco $\mathrm{P}$, Lamont $\mathrm{J}$ and Sawhney M. Persistent psychological or physical symptoms following endoscopic procedures: An unrecognized post endoscopy adverse event, Dig Dis Sci J. 2010; 55: 286973.

23. Essink-Bot M, Kruijshaar M and Bac D. Different perceptions of the burden of upper GI endoscopy: An empirical study in three patient groups, Qual Life Res J. 2007; 16: (8)1309-18.

24. Bennett S, Gostimir M and Shorr R. The role of routine postoperative upper endoscopy in bariatric surgery, Surg Obes Relat Dis J. 2016; 10:755-65.

25. Yoenlee J, Anhn M, Kim D and Kweon $\mathrm{H}$. The effect of preparatory education program on discomfort and 
retching of examinees during upper gastrointestinal endoscopy, Korean J Fam Med. 2012; 33(4): 219-28. Available at: http://synapse.koreamed.org.

26. Maguire D, Walsh C and Little L. The effect of information and behavioral training on endoscopy patient's clinical outcome, Patient Education and Counseling J. 2004; 54(1):61-5.

27. Adang R, Vismans $J$ and Talmon $J$. Appropriateness of indications for upper gastrointestinal endoscopy: Associations with relevant endoscopic disease, Gastrointest Endosc J. 2010; 42:390-7.

28. Spielberger C, Gorsuch R and Lushene R. Handbook for the IPAT anxiety scale. 2nd ed. Institute for personality and ability testing. 1970.

29. El-Behairy R. State Trait Anxiety Inventory. Assessment of anxiety level in patients before diagnostic endoscopy. 1984.

30. Elsayd O, Elshemy M and Elsays $\mathrm{H}$. Effect of a multi-modal preparation package on knowledge and anxiety among patients undergoing cardiac catheterization, International Journal of Nursing Didactics. 2016; 6:1-10.

31. Tluczek A, Henriques $J$ and Brown R. Support for the reliability and validity of the state anxiety scale, J Nurs Meas. 2009;17:19-28.

32. Chiu J, Lee C, Lai Y, Yng R and Tu T. Identification of factors that impact on patient satisfaction of un sedated upper gastrointestinal endoscopy, Journal of Clinical Nursing. 2009; 2(4):280-9.

33. Sewitch M, Gong S, Barkun A, Hilsden R and Dube C. Literature review of quality in upper and lower gastrointestinal endoscopy from the patient perspective, Can J Gastroenterol. 2011; 25(1):681- 5 .

34. Ruhl E and Everhart J. Assess indications and outcomes needs of GI endoscopy. 2016; 3(1):1846.Availableat: http://www3.niddk.nih.

35. Lee Y, Anhn M, Kim E, Kweon H and Yoon M. The effect of preparatory education program on discomfort and retching of examinees during upper gastrointestinal endoscopy, Korean J Fam Med. 2012; 33(4): 219-28.

36. El said S. Effectiveness of structured teaching program on knowledge, anxiety state and tolerance for patients with gastrointestinal endoscopy: Randomized controlled trial, Life Science Journal. 2016; 13(9): 9-17.

37. Seda P, Nimet O, Mehmet K, Yavuz P and Murat T. Effect of providing information to the patient about upper 
gastrointestinal endoscopy on the patient's perception, compliance and anxiety level associated with the procedure, Turk J Gastroenter. 2011; 22 (1):10-7.

38. Thomas $\mathrm{S}$ and Sugirtha V. Effectiveness of structured teaching programme on knowledge and anxiety of patients undergoing endoscopy at a gastroenterology centre of a tertiary care hospital, International Journal of Science and Research. 2015; 4(6): 1017-20. Available at: www.ijsr.net.

39. Feyzullah E, Toros A, Aydo G, Bektafi $\mathrm{H}$ and Ozcani O. Assesment of anxiety levels in patients during elective upper gastrointestinal endoscopy and colonoscopy, Turk J Gastroenterol. 2010; 21(1):29- 33.

40. Mohamed S. Effectiveness of structured teaching program on knowledge, anxiety state and tolerance for patients with gastrointestinal endoscopy, Life Science J. 2016; 13(9):9-17. Available at: www.lifesciencesite.com.

41. Abdulla W. Assessment of knowledge and perception of the patient with upper gastrointestinal disease. Master Thesis, Faculty of Nursing, Ain Shams University. 2010.

42. Prabhuswami H, Vaishali $\mathrm{R}$ and
Prakash N. Knowledge, attitude and pre-procedure anxiety level of patient undergoing upper gastrointestinal endoscopy in Krishna Hospital, India End J. 2016; 5 (6):243-9.

43. Kennedy J, Oconnor P and Sadovnick A. Upper gastrointestinal diseases influenced by place of residence, American Journal of Nursing. 2006; 93 (9): 162-7.

44. Vliet M, Grypdonck M and Zuuren F. Preparing patients for gastrointestinal endoscopy: The influence of information in medical situations, Patient Educ Couns J. 2004; 52: 23-30.

45. Quick J. Social support, secure attachments and health. Handbook, Philadelphia, Lippincott. 2006; 2(3): 169-72.

46. Winslow E. Patient education materials in upper GI endoscopy, American J of Nursing. 2004; 102 (17): $33-8$.

47. Mohamad H, Mohamad Z and Sayed Z. Impact of a designed nursing teaching protocol on nurse's performance and patient's outcome in Esophagogastroduodenoscopy, Journal of American Science. 2014; 10 (10): 56-65.

48. Previti G, Bianchini O, Virzi A, Petralia A and Aguglia E. Identifying 
people at risk for anxiety in patients undergoing endoscopic procedures, Ann Depress Anxiety J. 2016; 3(1):1072-5.

49. Smith P. Traditions, trends, tomorrow. Gastroenterol Nurs J. 2002; 25(1):81-2. 50. Axon A, Jones $\mathrm{R}$ and Quine $\mathrm{M}$. Guidelines on appropriate indications for upper gastrointestinal endoscopy, American Nursing J. 2010; 313 (5): 922-8.

51. Yamada T. Endoscopy. Handbook of Gastroenterology. USA: Lippincott Williams \&Wilkins. 2002; 3(1): 667-80.

52. Classen M. Informed consent for gastroenterological endoscopy, Gastroenterological Endoscopy J. NewYork. 2002; 2(1):7-9.

53. Abuksis G, Mor M and Segal N. A patient education program is cost effective for preventing failure of endoscopic procedures in a gastroenterology department, American Gastroenterol J. 2001; 97(2):1786-90.

54. Chair S and Thompson D. Patient teaching prior to coronary angiography in Hong Kong: A pilot study, Clinical Nursing J. 2005; 14(10): 114-5.

55. Shepherd H, Bowman D and Hancock B. Postal consent for upper gastrointestinal endoscopy, Gutz J.
2000; 46(1): 37-9.

56. Manhas S, Vizet J, Deby S, Vanel J, Boito P, Verdier M, Martino A and Pagnoux D. Demonstration of full $4 \times 4$ Mueller polarimetry through an optical fiber for endoscopic applications, Optics Express J. 2015; 23(3): 304754.

57. Balci O, Acar A and Mahmoud A. Effect of providing information to the patient about upper gastrointestinal endoscopy on the patient's perception, compliance and anxiety level associated with the procedure, The Turkish Journal of Gastroenterology. 2011; 2 (22):18-20. Accessed on $15 / 8 / 2012$.

58. Deirdre M, Walsh J and Little C. The effect of information and behavioral training on endoscopy patients' clinical outcomes, Patient Education and Counseling J. 2004; 3:5461-5. Available at: www.nuigalway.psychology. Accessed on $18 / 8 / 2012$.

59. Eberhardt J, Wersch A and Cann P. Information, social support and anxiety before gastrointestinal endoscopy, Britch Health Psychology J. 2006; 11(4):551-9.

60. Morgan J, Roufeil L, Kaushik S and Bassett M. Influence of coping style 
and pre-colonoscopy information on pain and anxiety of colonoscopy. 2012; 48 (2):134-5. Available at: www.ncbi.nlm.nih. Accessed on /15/8/2014.

61. Spalding N. Reducing anxiety by preoperative education: Make the future familiar. Norwich, UK. 2003; 10(4):278-93 Available at: http://www.ncbi.nlm.nih.gov.

Accessed on: 14/08/2012.

62. Speilberger C. State-Trait Anxiety Inventory for adult, self-evaluation questionnaire STAI forms $\mathrm{Y}-1$ and $\mathrm{Y}$ 2. Redwood City, CA: Mind Garden, 1977.

63. Riddhiputra P and Ukarapol N. Effect of systematic psychological preparation using visual illustration prior to gastro intestinal endoscopy on the anxiety of both pediatric patients and parents, Med Assoc Thai J. 2006; 89(2): 231-5.

64. Hayes A, Buffum M, Lanier E, Rodahl $\mathrm{E}$ and Sasso C. A music intervention to reduce anxiety prior to gastrointestinal procedures, Gastroenterol Nurs J. 2003; 26(1): 145-9.

65. Jones $\mathrm{M}$, Ebert $\mathrm{C}$ and Solan $\mathrm{T}$ Relation between patient's anxiety and elective upper gastro endoscopy, Clinic Gastroenterol J. 2004; 38(1): 35 -
40.

66. Brandt L. Patients' attitudes and apprehensions about endoscopy: How to calm troubled waters. Americian Gastroenterol J. 2001; 96 (1): 280-4.

67. Trevisani L, Sartori S and Putinati S. Assessment of anxiety level in patients during diagnostic endoscopy. Recenti Prog Med J. 2002; 93(1): 240-4.

68. Essink-Bot M, Kruijshaar $M$ and Bac D. Different perceptions of the burden of upper GI endoscopy: An empirical study in three groups. Qual Life Res J. 2016; 16:1309-18.

69. Ajee K. A study to assess the effectiveness of structured teaching on anxiety and physiological responses of adult patients undergoing endoscopy procedure. MSc Nursing. College of Nursing CMC Vellore: Tamilnadu University 2002.

70. Aabakken L. Development and evaluation of written patient information for endoscopic procedure, Endoscopy J. 2010; 29: 23-6.

71. Kiyohara Y and Kayanolk M. Anxiety in preoperative anesthetic endoscopy procedure, Rev Hosp Clinic Med Sao Paulo J. 2004; 11 (2):51-6.

72. Rohde H. Anxiety before gastrointestinal endoscopy is a significant problem, Int End J. 2011; 
115(2):1539-44.

73. Peter B. Cotton N, Christopher B, Robert $\mathrm{H}$ and Brian $\mathrm{P}$. The Fundamental of upper GI endoscopy, Philadelphia: Lippincott Williams

74. Mulcahy $\mathrm{H}$ and Kelly P. Factors associated with tolerance to, and discomfort with unsedated diagnostic gastroscopy, Scand J Gastroentrol. 2010; 36:1352-7.

75. Hashimoto T, Adachi $\mathrm{K}$ and Ishimura N. Safety and efficacy of glucagon as a pre medication for upper gastrointestinal endoscopy: A comparative study with butyl scopolamine bromide, Aliment Pharmacol Ther J. 2002; 16(1): 111-8.

76. Lee D, Chan A and Wong S. Can visual distraction decrease the dose of patient-controlled sedation required during endoscopy? A prospective randomized controlled trial, Endoscopy J. 2004; 36: 197-201.

77. Campo R, Brullet E and Montserrat A. Identification of factors that influence tolerance of upper gastrointestinal endoscopy, Eur Gastroenterol Hepatol J. 2015; 21:210-24.

78. Muzzarelli L, Force M and Sebold M. Aromatherapy and reducing pregastrointestinal endoscopy anxiety: A controlled prospective study,
Gastroenterol Nurs J. 2006; 29: 466-71.

79. Hakan U, Gamze $\mathrm{O}$ and Haldun S. Departments of gastroenterology, Psychiatry and Internal Medicine J: Baflkent University School of Medicine, Ankara. 2012; 20(3): 67-71. 80. Ersoz F, Toros A and Aydogan G. Assessment of anxiety levels in patients during elective upper gastrointestinal endoscopy and colonoscopy, Turk Gastroenterol J. 2010; 21: 29-33 